\title{
COREFLEXIVE AND SOMEWHAT REFLEXIVE BANACH SPACES
}

\author{
JAMES R. CLARK ${ }^{1}$
}

\begin{abstract}
A coreflexive Banach space is shown to have many of the same properties as a quasi-reflexive space. An infinite dimensional reflexive subspace of a Banach space with boundedly complete basis and separable dual is constructed, and it is noted that somewhat reflexive Banach spaces need not be coreflexive.
\end{abstract}

0 . Introduction. Let $X$ be a Banach space and let $X^{*}$ and $X^{* *}$ denote the first and second conjugate spaces of $X$. If $Q$ denotes the canonical map of $X$ into $X^{* *}$, then [2] $X$ is quasi-reflexive of order $n$ if $\operatorname{dim} X^{* *} / Q(X)=n$. We say that $X$ is coreflexive if $X^{* *} / Q(X)$ is reflexive and that $X$ is complemented coreflexive if $Q(X)$ is complemented by a reflexive subspace of $X^{* *}$. A somenhat reflexive Banach space [7] is a Banach space in which each infinite dimensional closed subspace contains an infinite dimensional reflexive subspace. Herman and Whitley [7] give an example of a somewhat reflexive space which is coreflexive. In this paper we investigate the conjecture that a necessary and sufficient condition for somewhat reflexivity is coreflexivity (complemented coreflexivity). In $\$ 2$ we develop some properties of coreflexive spaces, many of which hold for quasi-reflexive spaces. In $\S 3$ we give a proof that every Banach space with boundedly complete basis and separable dual contains an infinite dimensional reflexive subspace. This theorem is a consequence of a theorem in [9], however our proof involves an interesting constructive process so we include it here. Using results of [9] we are able to prove that if $X$ is a Banach space such that $X^{* *} / Q(X)$ is separable, then $X$ and $X^{*}$ are somewhat reflexive. Finally we note that somewhat reflexive spaces need not be coreflexive and pose some unanswered questions.

Presented to the Society, November 28, 1970 under the title An example concerning somewhat reflexivity; received by the editors October 26,1971 .

AMS (MOS) subject classifications (1970). Primary 46B99; Secondary 46B05, 46B10, 46B15.

Key words and phrases. Reflexive Banach spaces, somewhat reflexive Banach spaces, coreflexive Banach spaces, boundedly complete basis, shrinking basis, unconditional basis, compact operator, total subspace, $V$-pseudo-reflexive.

${ }_{1}$ This paper includes some of the results from the author's doctoral dissertation written at the University of Missouri-Columbia, where the author was partially supported by an NDEA Fellowship. The author wishes to express his thanks to Dr. Richard J. Fleming for his aid and encouragement. 
1. Notation and definitions. If $X$ is a Banach space and $Q$ is the canonical isomorphism into $X^{* *}$, we let $H(X)=X^{* *} / Q(X)$. For $M$ a subset of $X, M^{0}$ will denote the annihilator of $M$ in $X^{*}$. If $M$ is a subset of $X^{*},{ }^{0} M$ denotes the annihilator of $M$ in $X$. A one-to-one, bicontinuous, linear map $T$ from $X$ onto $Y$ is called an isomorphism, and $X$ and $Y$ are said to be isomorphic, denoted $X \simeq Y$. If $T$ is also norm-preserving, $X$ and $Y$ are said to be congruent, and we write $X \equiv Y$. By "subspace" we will always mean "closed subspace." The closed unit ball of $X$ will be denoted $S_{X}$.

2. Coreflexive Banach spaces. We begin by obtaining some results which extend certain theorems of Civin and Yood [2] to coreflexive spaces.

2.1 THEOREM. Let $X$ be a Banach space and $M$ a closed subspace of $X$, then

(a) $X$ is coreflexive if and only if $M$ and $X / M$ are coreflexive,

(b) $X$ is coreflexive if and only if $X^{*}$ is coreflexive, and

(c) $H(X)$ is reflexive and separable if and only if $H\left(X^{*}\right)$ is reflexive and separable.

Proof. (a) Suppose $X$ is coreflexive. By [2, p. 908], $H(M) \simeq Q(X)+$ $M^{00} / Q(X)$ and $H(X / M) \simeq X^{* *} / Q(X)+M^{00}$. Since subspaces and quotients of reflexive spaces are reflexive, $M$ and $X / M$ are reflexive.

Conversely if $Q(X)+M^{00} / Q(X)$ and $X^{* *} / Q(X)+M^{00}$ are reflexive, then $H(X)$ is reflexive [6, p. 72].

(b) By [5, p. 1066], $Q\left(X^{*}\right)$ is complemented in $X^{* * *}$ by $Q(X)^{0}$. Since $H(X)^{*} \equiv Q(X)^{0} \simeq H\left(X^{*}\right)\left[6\right.$, p. 72] $H(X)$ is reflexive if and only if $H\left(X^{*}\right)$ is reflexive.

(c) The proof is immediate from the proof of (b) and the fact that a Banach space is reflexive and separable if and only if its dual is reflexive and separable.

By considering the proof of 2.1 , we also may conclude that for conjugate spaces, the notions of coreflexivity and complemented coreflexivity coincide. In fact a complemented coreflexive space is necessarily isomorphic to a conjugate space. The following theorem characterizes complemented coreflexive spaces.

Recall that by a total subspace $V$ of $X^{*}$, we mean a subspace such that ${ }^{0} V=\{0\}$. For $V$ a total subspace of $X^{*}$, we say that $X$ is $V$-pseudo-reflexive [13] if the map $T$ from $X$ into $V^{*}$ defined by $(T x) f=f(x)$ for $f$ in $V$ is an isomorphism onto. We let $\sigma(X, V)$ denote the weakest linear topology on $X$ that makes each $f$ in $V$ continuous. 
2.2 THEOREM. Let $X$ be a Banach space. The following are equivalent.

(a) $X$ is complemented coreflexive.

(b) There exists a total subspace $V$ of $X^{*}$ such that $X^{*} / V$ is reflexive and an equivalent norm $|\mathrm{l} \cdot| \mathrm{I}$ on $X$ such that $S_{X}$ is $\sigma(X, V)$ compact.

(c) There exists a total subspace $V$ of $X^{*}$ such that $X$ is $V$-pseudoreflexive.

(d) There exists a total closed subspace $V$ of $X^{*}$ such that $X^{*} / V$ is reflexive and $S_{X}$ is $\sigma(X, V)$ conditionally compact.

Proof. (a) $\rightarrow$ (b). If $E$ complements $Q(X)$ in $X^{* *}, E$ must be $\sigma\left(X^{* *}, X^{*}\right)$ closed [14, p. 232]. Thus $V={ }^{0} E$ is a total subspace of $X^{*}$ and $\left(X^{*} / V\right)^{*} \equiv E$ is reflexive [14, p. 232]. By [5, pp. 1064-1066], $X$ may be given an equivalent norm such that $S_{X}$ is $\sigma(X, V)$ compact.

(b) $\rightarrow$ (c) $\rightarrow$ (d). [13, p. 77].

(d) $\rightarrow$ (a). If $S_{X}$ is conditionally $\sigma(X, V)$ compact, $V^{0}$ complements $Q(X)$ [5, pp. 1065-1066]. Since $V^{0} \equiv\left(X^{*} / V\right)^{*}, V^{0}$ is reflexive.

We note that we do not know if subspaces of complemented coreflexive spaces are complemented coreflexive. However, complemented subspaces of complemented coreflexive spaces are necessarily complemented coreflexive. This follows from the fact that complemented subspaces of a space complemented in its second conjugate space are themselves complemented in their second conjugate. Since such subspaces are necessarily coreflexive, they must necessarily be complemented coreflexive.

The question arises whether $X$ coreflexive implies that $X$ is complemented coreflexive. An affirmative answer to this question would imply that a coreflexive space is necessarily a conjugate space of all orders, a property enjoyed by quasi-reflexive spaces [2]. Indeed if this were the case, coreflexivity would be sufficient for somewhat reflexivity. For if $M$ is an infinite dimensional subspace of $X$, we have $M \simeq Y^{* *}$ for suitable $Y$. If $Y$ is quasi-reflexive. $M$ contains an infinite dimensional reflexive subspace [7, p. 291]. If $Y$ is not quasi-reflexive, the infinite dimensional reflexive complement of $Y$ provides the desired subspace.

We note that an example of a non-quasi-reflexive space which is a conjugate space of all orders is the $l^{2}$ sum $[4$, p. 31] of a quasi-reflexive space. This example is also somewhat reflexive [7, p. 293].

2.3 THEOREM. A coreflexive Banach space can contain no subspaces isomorphic to $c_{0}, c$ or any of their conjugates. In particular, a coreflexive Banach space with an unconditional basis is reflexive.

Proof. Any subspace of a coreflexive space is coreflexive, however neither $c_{0}$ nor any of its conjugates are coreflexive. To see this, assume $c_{0}$ is coreflexive, and thus $c_{0}^{*}=l^{1}$ is also coreflexive. Now $c_{0}^{*}$ is complemented 
in $m^{*}$, so there is a continuous projection of $m^{*}$ onto $l^{1}$. But $H\left(l^{1}\right)$ is infinite dimensional, contradicting $\left[\mathbf{1 5}\right.$, p. 253]. Thus $c_{0}$ cannot be coreflexive, and $c$, which is isomorphic to $c_{0}$, cannot be coreflexive.

If a Banach space with an unconditional basis contains no subspace isomorphic to $c_{0}$ or $l^{1}$, it is reflexive [4, p. 74]. In particular a coreflexive Banach space with an unconditional basis is reflexive.

We conclude this section with a theorem which has been proved for both quasi-reflexive [2] and somewhat reflexive spaces [7].

2.4 TheOREM. Let $T$ be a continuous linear map from a coreflexive space $X$ into $I^{1}$; then $T$ is compact.

Proof. If $T$ is a bounded linear map from $X$ into $l^{1}, T^{*}$, the adjoint of $T$, is a bounded map from $l^{*}$ to $X^{*}$. By 2.1 and 2.3, $T^{*}$ cannot have bounded inverse on any subspace isomorphic to $c_{0}$. Since $\left(l^{1}\right)^{*}=m$ may be viewed as a $C(S)$-space, it follows that $T^{*}$ must be weakly compact [12, p. 35]. Thus $T$ is weakly compact by Gantmacher's theorem [6]. In $l^{1}$, however, weak and norm convergence of sequences are the same [4, p. 33], so $T$ is compact.

3. Somewhat reflexive spaces. The next theorem and its corollary are special cases of [9, Theorem 4.2]; however, our proof involves an interesting constructive process so we have included it here.

3.1 THEOREM. If $X$ is a Banach space with boundedly complete basis and separable dual, $X$ has an infinite dimensional reflexive subspace.

Proof. It suffices [4, pp. 67-70] to show that a Banach space $Y$ with shrinking basis and separable second conjugate space has an infinite dimensional reflexive quotient.

If $\left(\left[F_{i}\right]\right)$ is a dense sequence of cosets in $H(Y)$, we may choose a maximal linearly independent subsequence $\left(\left[H_{i}\right]\right)$. Now $\left(Q\left(b_{i}\right)\right)$ forms a $w^{*}$-basis for $Y^{* *}$. so we may assume that each representative $H_{i}$ is given by

$$
H_{i}={ }_{r *} \sum_{j=1}^{\infty} a_{i, j} Q\left(b_{j}\right) \quad \text { and } \quad a_{i, j}=0, \quad 1 \leqq j \leqq 2^{i} .
$$

Define a sequence $\left(h_{i, k}\right)$ by

$$
\begin{aligned}
& h_{i, 1}=a_{i, 1} Q\left(b_{1}\right)+a_{i, 2} Q\left(b_{2}\right) \quad \text { and } \\
& h_{i, k}=\sum_{j=2^{k=1} \div 1}^{2^{k}} a_{i, j} Q\left(b_{i}\right), \quad k=2,3, \cdots .
\end{aligned}
$$

By (1) we note that $h_{i, k}=0$ for $k \leqq i$. Also, $H_{i}={ }_{w^{*}} \sum_{k=1}^{\infty} h_{i, k}, i=1,2, \cdots$. 
Let $M$ be the closed linear span of $\left(h_{i, k}\right)$ in $Y$. For any integers $n$ and $i$ such that $2^{n}+1 \leqq i \leqq 2^{n+1}$, it follows that $b_{i}$ is in $M$ if and only if there exists a sequence $\left(d_{i, m, n}\right)$ of scalars such that

$$
\lim \left[\begin{array}{ccc}
a_{1,2^{n}+1} & \cdots & a_{n, 2^{n}+1} \\
\cdot & & \cdot \\
\cdot & & \cdot \\
\cdot & & \cdot \\
a_{1,2^{n+1}} & \cdots & a_{n, 2^{n+1}}
\end{array}\right]\left[\begin{array}{c}
d_{i, m, 1} \\
\cdot \\
\cdot \\
\cdot \\
d_{i, m, n}
\end{array}\right]=\left[\begin{array}{c}
0 \\
\cdot \\
1 \\
\cdot \\
0
\end{array}\right]
$$

where the matrix on the right in (3) has all zero entries except a 1 in the $\left(i-2^{n}\right)$ th row. It is easy to see that if scalars exist such that (3) holds for all integers $i, 2^{n}+1 \leqq i \leqq 2^{n+1}$, then the coefficient matrix must have rank $2^{n}$. Since this is an obvious contradiction, it follows that for each positive integer $n$, there is at least one integer $i, 2^{n}+1 \leqq i \leqq 2^{n+1}$ such that $b_{i}$ is not in $M$. Given a positive integer $n$, choose one such $b_{i_{n}}$. We claim that $\left(\left[b_{i_{n}}\right]\right)$ is linearly independent in $Y / M$. For if $[0]=\sum_{k=1}^{i_{n}} a_{k}\left[b_{i_{n_{k}}}\right]$, then $b_{i_{n_{1}}}=-1 / a_{1}\left(\sum_{k=2}^{p} a_{k} b_{i_{n}}\right)+m$, for some $m$ in $M$. Let $\left(B_{i}\right)$ be the sequence of linear functionals such that $B_{i}\left(b_{j}\right)=\delta_{i, j}$. For $t=i_{n_{1}}$, some $q$, and all $j$ such that $2^{q}+1 \leqq t, j \leqq 2^{q+1}$, we have $\delta_{j, t}=B_{j}\left(b_{t}\right)=\lim _{m} \sum_{k=1}^{q} d_{t, m, k} a_{k, j}$ for a suitable sequence of scalars $\left(d_{t, m, k}\right)$, contradicting the choice of $b_{t}(3)$. Thus $Y \mid M$ is infinite dimensional.

To show that $Y / M$ is reflexive, we show that $Q(Y)+M^{00}$ is dense in $Y^{* *}$ [2, p. 908]. Since each $H_{i}$ is in $M^{00}[14$, p. 232], it follows that every $F_{i}$ is in $Q(Y)+M^{00}$. Because $\left(\left[F_{i}\right]\right)$ is dense in $H(Y), Q(Y)+M^{00}$ is seen to be dense in $Y^{* *}$.

3.2 Corollary. A Banach space with boundedly complete basis and separable dual is somewhat reflexive.

Proof. Any infinite dimensional subspace $M$ contains a subspace isomorphic to a subspace with a block basis [1, p. 157]. Such a basic sequence is necessarily boundedly complete. By Theorem 3.1, $M$ contains an infinite dimensional reflexive subspace.

The proof of Theorem 3.1 relies on (1) the separability of $H(Y)$ and (2) the fact that $\left(Q\left(b_{i}\right)\right)$ forms a $w^{*}$-basis for $Y^{* *}$. One can easily see that the construction fails if we do not demand that $X^{*}$ be separable as, for example, $l^{1 *}$.

Johnson and Rosenthal [9] prove the theorem: If $X^{* *}$ is separable then both $X$ and $X^{*}$ are somewhat reflexive. The next theorem extends this result to nonseparable Banach spaces.

3.3 Theorem. Let $H(X)$ be separable. Then $X$ and $X^{*}$ are somewhat reflexive. 
Proof. It suffices to show that separable subspaces of $X$ and $X^{*}$ contain reflexive subspaces. If $M$ is a separable subspace of $X, M^{* *}$ is separable [11, p. 121]. By [9], $M$ contains a reflexive subspace. If $M$ is a separable subspace of $X^{*}$, we may consider a dense sequence $\left(f_{n}\right)$ in $M$. For each $n$, there is a sequence $\left(x_{n, i}\right)$ in $S_{X}$ such that $\lim _{i}\left|f_{n}\left(x_{n, i}\right)\right|=\left\|f_{n}\right\|$. If $N$ is the closed linear span of $\left(x_{n . i}\right)$, the restriction of $f$ in $M$ to $N$ is an isometry of $M$ into $N^{*}$. Since $N^{* *}$ is separable [11, p. 121] and $M$ is isomorphic to a subspace of $N^{*}$, the result follows from [9].

We note that the converse to Theorem 3.3 is false. If $S$ is an uncountable set and if $X$ is the $l^{2}-(S)$-sum [4, p. 31] of a quasi-reflexive space of order $1, X$ is somewhat reflexive. This is a consequence of the fact that separable subspaces of $X$ can be considered as a subspace of an $l^{2}$-sum of quasi-reflexive spaces, which is somewhat reflexive [7, p. 293]. However, $H(X)$ is not separable.

3.4 THEOREM. If $H(X)$ is reflexive and separable, each conjugate of $X$ is somewhat reflexive.

Proof. If $Y$ is a conjugate of $X, H(Y)$ is reflexive and separable (2.1). Theorem 3.4 implies that $Y$ is somewhat reflexive.

3.5 ExAmple. A somewhat reflexive space need not be coreflexive (complemented coreflexive). If $X$ is the space $B_{3}^{*}$ constructed in [8] and [10], $B_{3}^{*}$ is somewhat reflexive, but $H\left(B_{3}^{*}\right) \simeq m$.

It was noted in [3] that quotients and duals of somewhat reflexive spaces need not be somewhat reflexive. The question is open whether $X^{*}$ somewhat reflexive implies that $X$ is somewhat reflexive. However, it is interesting to note that there exist Banach spaces $X$ and $Y$ such that $X^{*} \simeq Y^{*}$ and $X$ is somewhat reflexive but $Y$ is not somewhat reflexive. For example, let $X=B_{3}^{*}$ [8] and $Y=B_{3}^{*} \oplus c_{0}$. Since $X^{*}=B_{3} \oplus /^{1}$ and $Y^{*}=$ $B_{3} \odot l^{1} \ominus l^{1}, X^{*}$ and $Y^{*}$ are isomorphic. Now $Y$ contains $c_{0}$, so $Y$ cannot be somewhat reflexive.

We conclude with some unanswered questions.

(1) If $X$ is separable and coreflexive, is it the case that $H(X)$ is separable?

(2) Does every non-somewhat reflexive space contain a subspace isomorphic to $c_{0}$ or $l^{1}$ ?

If the answer to either of these questions is affirmative, coreflexivity implies somewhat reflexivity. Finally we ask:

(3) Every quasi-reflexive space is a dual. Is every somewhat reflexive (coreflexive) space a dual?

\section{REFERENCES}

1. Cz. Bessaga and A. Pelczyński, On bases and unconditional convergence of series in Banach spaces, Studia Math. 17 (1958), 151-164. MR 22 \#5872.

2. P. Civin and B. Yood, Quasi-reflexive spaces, Proc. Amer. Math. Soc. 8 (1957), 906-911. MR 19, 756. 
3. W. Davis and I. Singer, Boundedly complete M-bases and complemented subspaces in Banach spaces (to appear).

4. M. M. Day, Normed linear spaces, 2nd ed., Ergebnisse der Mathematik und ihrer Grenzgebiete, N. F., Heft 21, Academic Press, New York; Springer-Verlag, Berlin, 1962. MR 26 \#2847.

5. J. Dixmier, Sur un théorème de Banach, Duke Math. J. 15 (1948), 1057-1071. MR 10, 306.

6. N. Dunford and J. T. Schwartz, Linear operators. I: General theory, Pure and Appl. Math., vol. 7, Interscience, New York, 1958. MR 22 \#8302.

7. R. Herman and R. Whitley, An example concerning reflexivity, Studia Math. 28 (1966), 289-294. MR 35 \#5900.

8. R. C. James, Separable conjugate spaces, Pacific J. Math. 23 (1967), 113-117.

9. W. B. Johnson and H. P. Rosenthal, On $w^{*}$-basic sequences and their applications to the study of Banach spaces, Studia Math. 43 (1972), 77-92.

10. J. Lindenstrauss, On James' paper "Separable conjugate spaces," Israel J. Math. 9 (1971), 279-284.

11. R. D. McWilliams, On certain Banach spaces which are $w^{*}$-sequentially dense in their second duals, Duke Math. J. 37 (1970), 121-126. MR 41 \#4208.

12. A. Pelczyński, On strictly singular and strictly cosingular operators. I. Strictly singular and strictly cosingular operators in $C(S)$-spaces, Bull. Acad. Polon. Sci. Sér. Sci. Math. Astronom. Phys. 13 (1965), 31-36. MR 31 \#1563.

13. I. Singer, Weak compactness, pseudo-reflexivity and quasi-reflexivity, Math. Ann. 154 (1964), 77-87. MR 29 \#2633.

14. A. E. Taylor, Introduction to functional analysis, Wiley, New York; Chapman \& Hall, London, 1958. MR 20 \#5411.

15. R. J. Whitley, Strictly singular operators and their conjugates, Trans. Amer. Math. Soc. 113 (1964), 252-261. MR 31 \#1565.

Department of Mathematics, University of MississipPI, University, MississipPi 38677 